And now I have the great honor to present the first album of initial photographs of the Moon taken by the Ranger spacecraft to the Director of the Observatory of our host University, Prof. Haffner. Other albums will be distributed to Directors of the major observatories throughout the world.

The first paper will be presented by Mr Schurmeier, Project Manager, who bore the responsibility for its operation with broad and capable shoulders.

\title{
2. THE RANGER VII MISSION
}

H. M. Schurmeier, R. L. Heacock

(Jet Propulsion Laboratory, California Institute of Technology, Pasadena, California, U.S.A.)

and

B. P. Miller

(Astro-Electronics Division, Radio Corporation of America, Princeton, New Jersey, U.S.A.)

The Ranger system uses an attitude-stabilized spacecraft to get the advantages of maximum solar power generation; maximum communications capability; spacecraft-centered coordinate system for use in midcourse maneuvers; and direction and control capabilities for accurate scientific instrument observations. Ranger also utilizes the parking orbit technique, permitting maximum payload to be efficiently injected into the desired trajectory.

In spite of the Ranger Blocks I and II launch vehicle and spacecraft system problems, valuable knowledge and experience was gained and applied to Mariner II and Rangers VI and VII. The results of these missions included an extremely successful fly-by of Venus and two lunar impacts of pinpoint accuracy.

Block III of the Ranger program differs from Block II only in the payload which consists of fast sequence-shuttered TV cameras operating into two high-power transmitters.

Ranger VI failed to return pictures because of power supply destruction due to unscheduled turn-on of the TV system during launch.

The Atlas D/Agena B multistage launch vehicle was employed for the mission. Major constraints on the flight plan were (a) Earth-Moon geometry must be such that the spacecraft has adequate orientation accuracy during flight; $(b)$ lunar lighting conditions must provide contrast for good pictures; $(c)$ lunar encounter must be effected during Goldstone view period.

Launch window is about 2 hours per day of a 6-day launch period each month.

The spacecraft itself is hexagonal in shape. Power is supplied by both batteries and solar panels. Telecommunication subsystem includes antenna, radio, command and telemetry subsystems. The Guidance and Control system consists of central computer and sequencer and the autopilot and attitude control units. Propulsion for the midcourse maneuver is a monopropellant system using hydrazine. Temperature control is achieved through passive techniques. Explosive actuators are used in deploying solar panels and controlling the midcourse motor.

Six cameras, associated control and video circuitry, power system, and transmitters make up the TV subsystem. The two basic camera lenses are a $25 \mathrm{~mm} \mathrm{f/I} \cdot 0$ and a $76 \mathrm{~mm} \mathrm{f/2 \cdot 0}$. Photo- 
graphs are alternately taken by a $76 \mathrm{~mm}$ lens and a $25 \mathrm{~mm}$ lens. The vidicon is a specially designed rugged tube made by Radio Corporation of America.

The ground complex is the Deep Space Network and it includes $(a)$ three tracking stations located in California, South Africa, and Australia; $(b)$ the Space Flight Operations Facility located at the Jet Propulsion Laboratory in California; and (3) world-wide voice and teletype communications network. TV signals from the spacecraft are separated and recorded by the ground system on devices similar to commercial kinescopes used for filming TV programs. The signals are also recorded on magnetic tape.

Three highly-trained and specialized groups necessary to a space mission are (a) Spacecraft Data Analysis Team; (b) Flight Path Analysis and Command Group; and (3) Space Science Analysis Group.

All flight events occurred in normal sequence and at the proper time. A midcourse maneuver was performed which placed the impact only 6 miles from the target of $I^{\circ}$ south and $2 I^{\circ}$ west lunar selenocentric coordinates. No terminal orientation maneuver was required. The camera system returned over 4300 excellent photographs of the Moon during its $I_{7}$ minutes of operation prior to impact. The last photograph was taken at an altitude of approximately 500 meters. A surface resolution of approximately 0.5 meters was achieved. Cratering phenomena can be observed down to the limit of detectability in the photograph.

The tracking accuracy for Ranger VI and VII permitted refinement of the determination of the mass of both the Earth and the Moon.

Future Ranger missions will sample other mare areas to provide more information for the unmanned and manned lunar landing missions and increase our scientific knowledge of the Moon.

This paper presents the results of research carried out under contract NAS 7-Ioo sponsored by NASA.

\title{
3. RANGER VII CAMERA CALIBRATION AND PERFORMANCE
}

\author{
G. M. Smith, D. E. Willingham, W. H. Kirhofer \\ (Jet Propulsion Laboratory, California Institute of Technology, Pasadena, \\ California, U.S.A.)
}

This paper discusses factors which increase usefulness of the existing Ranger VII photographic system design.

A mathematical model utilizing a camera system description; a generalized lunar photometric function; a human observer model; and a trajectory/spacecraft description was developed and programed into a digital computer for the purpose of selecting optimum lunar aiming areas. The output of the program was considered a performance indicator (an estimate of the smallest surface feature observable on $35 \mathrm{~mm}$ film positive from the camera system). The specific subjects considered in the computer program analysis are discussed in the paper in detail.

Several problems related to the photographic system itself, including light transfer characteristic, modulation transfer function, and system noise, are described and solutions given.

A comprehensive description of the complete Ranger VII mission is presented with emphasis on the measures taken to ensure a photographic success. Specific figures are given for all significant data connected with each phase of the mission from launch to impact.

This paper presents the results of research carried out under contract NAS 7-IOo sponsored by NASA. 